\title{
Conditions and Performance of Ideal RAKE Reception for Ultra-Wideband Signals in Lognormal Fading Channels
}

\author{
J. Zhang, ${ }^{1,2}$ R. A. Kennedy, ${ }^{1}$ T. D. Abhayapala ${ }^{1}$
}

\begin{abstract}
In this paper, the conditions and performance of ideal RAKE reception for time hopping UltraWideband (UWB) investigated. Owing to the complex propagation phenomena and specific structure of UWB signals, new problems relevant to the operation of RAKE receivers arise. This motivates us to reconsider the conditions under which a RAKE receiver can work effectively with negligible interference between fingers. Key findings are that the conditions not only relate to the property of time hopping codes, but also modulation methods. An analytical technique is introduced to derive explicit expressions of RAKE performance for various combining methods for a lognormal fading channel. Numerical results show that RAKE reception can largely improve the performance, and equal gain combining has comparable performance to maximum ratio combining.
\end{abstract}

KEY WORDS: Ultra-wideband; RAKE receiver; lognormal distribution.

\section{INTRODUCTION}

In the last few years, there has been a rapidly growing interest in Ultra-Wideband (UWB) systems [1-4]. Combined with spread spectrum techniques, especially time hopping $(\mathrm{TH})$, ultra narrow pulses $(<1 \mathrm{~ns})$ are modulated to convey information in UWB. To avoid interference with existing wireless systems, the radiation power is restricted to be very low. RAKE reception [5] can collect multipath signals, and there exist many resolvable multipath components in UWB channels; thus a RAKE receiver could potentially be very helpful to UWB. But is it applicable and effective?

Some new problems relevant to the operation of RAKE reception arise because of the complex propagation phenomena of UWB signals. Firstly, in the spread

\footnotetext{
${ }^{1}$ National ICT Australia (NICTA) and the Department of Telecommunications, Research School of Information Science and Engineering, The Australian National University, Canberra ACT 0200, Australia.

22E-mail: jian@syseng.anu.edu.au.
}

spectrum context, a RAKE receiver takes advantage of the excellent autocorrelation performance of spread spectrum codes to eliminate the interference between fingers, such as interchip interference (ICI) and intersymbol interference (ISI). So the finger spacing is generally assumed to equal the chip width guided by sampling theory. In contrast, for TH-UWB, it is impractical to make a similar assumption because of the cluster structure observed in many field experiments [2,6-8] and the fact that the TH chip width is not necessarily the reciprocal of signal's bandwidth. Instead, we face a dense multipath channel with randomly arriving paths. For such a model, without the careful design of TH codes, the ICI will become significant and deteriorate the performance of a RAKE receiver. Second, because each multipath has very low energy, it is difficult to accurately estimate the exact amplitude. Further, the estimation error needs be considered when choosing a combining method, and combining methods that require low accuracy of estimates are preferred. Third, the number of resolvable multipaths with similar energy is very large because of the ultra-high multipath resolution ability of UWB pulses, and a good tradeoff between complexity and 
performance is required. These problems bring new challenges to the design of RAKE receivers. On the other hand, in the design of RAKE receivers, how to incorporate the properties of UWB pulses and the particular structure of TH signals, especially the low duty cycle, is worthy of further research.

In this paper, in consideration of these problems, we discuss conditions under which a RAKE receiver can operate ideally, that is, with negligible ICI in the output of each finger (hereafter, referred to as Clean output), as has not been studied systematically in the literature. We will show that, in most cases, it depends not only on the TH codes, but also on the modulation methods. For an adequate modulation method, only when the number of assignable chip positions in a frame (the maximum of $\mathrm{TH}$ codes as well) $\left(N_{c}\right)$ is comparable to the number of frames in a symbol period $\left(N_{f}\right)$ can satisfactory autocorrelation to mitigate ICI be achieved. It is also emphasized that ISI can be reduced in TH-UWB systems. These observations are based on a single user system; an extension to multiple user case is briefly discussed.

With the conditions highlighted, we further provide a method to evaluate the performance of ideal RAKE reception. Previous methods to evaluate the performance of a RAKE receiver for UWB are constrained to experimental ones [1,9] because of the absence of widely accepted fading channel models, especially the amplitude distribution, which is critical to performance analysis. Empirical distribution of the amplitude fading markedly differs from Rayleigh fading [2]. This is possibly because UWB signals have high resolution and only a small number of multipath components fall within an interval of delay resolution. In contrast, Rayleigh fading is justified directly from the complex Gaussian assumption based on the Central Limit Theorem, which requires large numbers of independent random variables. From the latest results generated by IEEE $802.15 \mathrm{sg} 3$ [2], the lognormal distribution is recommended to best fit the measured amplitude fading in a UWB multipath channel. So a time-delayed line (TDL) model with lognormal distributed amplitude is adopted in this paper.

Generally, exact performance evaluation of diversity reception is hardly tractable because of the complexity of the statistics. Fortunately, for the lognormal distribution in UWB applications, there are simplifications that make this tractable based on the fact that the sum of lognormal variables can be well approximated by another lognormal variable under appropriate conditions [10-12]. With the aid of Hermite-Gauss quadrature [13], explicit expressions of performance then can be obtained for the various combining methods such as equal gain combining (EGC) and maximum ratio combining (MRC). Numerical results show that EGC has comparable performance to MRC. This alleviates the channel estimation problem greatly.

The outline of this paper is as follows. Section 2 introduces the system model. Section 3 focuses on the conditions under which clean RAKE output can be achieved. Section 4 provides a method to analyze the performance of ideally operated RAKE receivers in a lognormal fading channel. Numerical results are shown in Section 5. Finally, some conclusions are given.

\section{MULTIPATH CHANNEL MODELS}

A UWB RAKE receiver consists of a group of RAKE fingers. Each finger, containing a bank of correlators and a combining weight chosen via combining criteria, synchronizes to a selected multipath. The performance and complexity of a RAKE receiver are largely influenced by the structure of multipath channels.

In [2], clusters and rays are used to account for the cluster phenomena in UWB propagations and a double set of parameters are used accordingly. This model presents difficulties for evaluating RAKE performance analytically because of the use of random Poisson process to simulate the arrival of rays and clusters and the resulting indeterminate mean power distribution of every path. To make analysis tractable, we simplify it to a TDL model that can be represented by the impulse response:

$$
h(t)=\sum_{\ell=1}^{L} a_{\ell} \delta\left(t-\tau_{\ell}\right)+n(t),
$$

where $n(t)$ is the additive white Gaussian noise (AWGN) with zero mean and variance $\sigma_{0}^{2}, \tau_{\ell}$ is the multipath delay, the fading amplitude $a_{\ell}$ is real, and its absolute value is lognormal distributed. When $20 \log _{10}\left|a_{\ell}\right|$ is Gaussian distributed with mean $u_{\ell}$ and variance $\sigma$, the probability density function (pdf) of $\left|a_{\ell}\right|$ is:

$$
\begin{array}{r}
p\left(\left|a_{\ell}\right|\right)=\frac{20}{\ln (10) \sqrt{2 \pi} \sigma\left|a_{\ell}\right|} \\
\exp \left(-\frac{\left(20 \log _{10}\left|a_{\ell}\right|-u_{\ell}\right)^{2}}{2 \sigma^{2}}\right),
\end{array}
$$

where $|\cdot|$ stands for absolute value. According to [2], $\sigma$ is fixed and $u_{\ell}$ varies with $\ell$. This distribution is denoted by $\left|a_{\ell}\right|: \Lambda\left(u_{\ell}, \sigma^{2}\right)$ where $\Lambda$ stands for lognormal distribution. The first and second moments of $\left|a_{\ell}\right|$ are given by:

$$
\begin{aligned}
\mathrm{E}\left[\left|a_{\ell}\right|\right] & =\exp \left(\ln (10) / 20 u_{\ell}+(\ln 10)^{2} / 800 \sigma^{2}\right), \\
\mathrm{E}\left[a_{\ell}^{2}\right] & =\exp \left(\ln (10) / 10 u_{\ell}+(\ln 10)^{2} / 200 \sigma^{2}\right) .
\end{aligned}
$$


As we will see in Section 4.T, the sum of two independent lognormal variables can be well approximated by another lognormal variable. This ensures that the cluster-plus-ray model proposed in [2] can be well approximated by the model suggested here even when overlapping of rays in different clusters happens. The remaining problem here is how to model the mean power of $a_{\ell}$. Two methods are considered. The first one, employing a general single exponential decay, is widely used in the literature, but it does not reflect the dual exponential fading model in [2] well. This distribution can be expressed as:

$$
\mathrm{E}\left[a_{\ell}^{2}\right]=\varepsilon_{0} \exp \left(-\tau_{\ell} / \Gamma\right),
$$

where $\varepsilon_{0}$ is the mean energy of the first arriving path $\left(\tau_{1}=0\right), \Gamma$ is the path decay factor. Then $u_{\ell}$ can be expressed as:

$$
u_{\ell}=10 / \ln (10)\left(\ln \varepsilon_{0}-\tau_{\ell} / \Gamma\right)-\ln (10) / 20 \sigma^{2} .
$$

The second one, specific to the model in [2], uses the mean power from experimental data generated in [2] directly.

\section{CONDITIONS FOR CLEAN RAKE OUTPUT}

A key condition in which a conventional RAKE works effectively is when ICI and ISI can be ignored. Ideal autocorrelation performance of spread spectrum (SS) codes is always assumed and utilized to eliminate the ICI. Note that ISI cannot be reduced in this way in most existing SS systems such as the widely used DS-CDMA. This is because the orthogonality of SS codes' autocorrelation could be destroyed by ISI unless the excess multipath delay is far smaller than the length of SS codes.

For TH-UWB, if the maximum multipath delay is as small as the frame period, ICI can be constrained to a small degree through deliberate adjustment of time-related system parameters because of the low duty cycle of UWB signals. This rarely happens in high-speed applications. In most cases, ideal autocorrelation and cross correlation of TH codes are required to reduce ICI.

There is little published work on TH codes to date. Two papers $[3,4]$, referring to the design of FH codes, discuss the method based on permutation sequences. It is indicated that the autocorrelation of TH codes is some kind of coincidence autocorrelation and equal to the number of overlapped positions [3,4] (we restrict ourselves to the case of pure TH codes where no amplitude information of codes is utilized). It is emphasized that the maximum autocorrelation is highly related to the relationship between the assignable chip positions in a frame $\left(N_{c}\right)$ and the period of TH codes $\left(N_{f}\right.$, which is also the number of pulses used to represent a symbol normally). Only when $N_{c}$ is not much smaller than $N_{f}$, can TH codes have desirable autocorrelation property. On the contrary, when $N_{f} \gg N_{c}$, designing TH codes with good autocorrelation is hardly possible.

Besides good autocorrelation, TH codes with $N_{c}$ comparable to $N_{f}$ can also lead to excellent cross correlation between different users. This configuration will not influence the multiuser capacity because multiple access ability is only constrained by the product of $N_{f}$ and $N_{c}$ but not by them separately in consideration to the number of assignable $\mathrm{TH}$ codes.

For TH-UWB, ideal autocorrelation is not the only necessary condition to ensure ICI to be eliminated; modulation methods also determine the effect of canceling ICI, a factor often ignored without justification. Poorly chosen modulations will cause similar decision variables to be generated by desired symbols and nondesired modulated symbols as a result of the correlation operation with multipath components. When the time jitter caused by modulations is smaller than the pulse width, this interference can be eliminated totally. This is based on the fact that the multipath resolution is generally larger than the width of pulse. The standard modulations combined with TH are $M$-ary PPM, antipodal (bipolar) modulation, amplitude modulation, and variations of them. Among them, orthogonal PPM (OPPM) will lead to a large decision error because of the potential ICI among modulated symbols. This is interpreted in detail from Figure 1. In Figure $1, p_{i m}$ stands for the $m$ th multipath signal of the $i$ th transmitted pulse in a symbol. Consider a simple binary-OPPM system as an example and suppose $T_{d}$ is the distance between the two OPPM symbols. If it happens that the distance between $p_{i 1}$ and $p_{i 2}$ equals $T_{d}$, when a RAKE finger is applied to $p_{i 1}$, the energy of $p_{i 1}$ and $p_{i 2}$ will be collected by the correlators corresponding to the two symbols, respectively. In slow fading channels, all pulses in a symbol period suffer the same fading so that the nondesired symbol will collect the energy of all pulses with the same index $m$. When many multipath

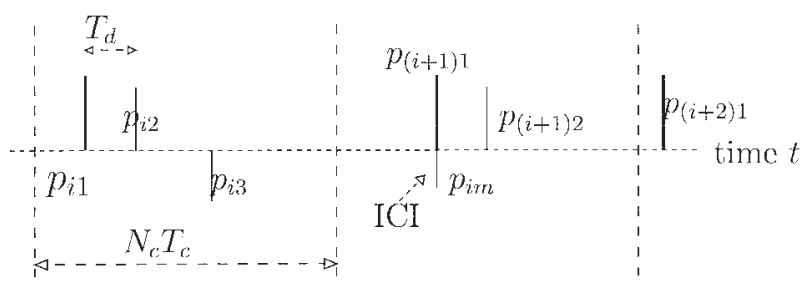

Fig. 1. Illustration of multipath signals and interference in THUWB systems; every solid line with the symbol $p(.$.$) represents a$ multipath signal. 
components have the distance $T_{d}$ between them, the nondesired symbol will collect enough energy and lead to high decision error. So OPPM is not applicable to TH-UWB without further design considerations. For overlapped PPM, the optimal modulation parameter is smaller than the width of a pulse because the values of the pulse's autocorrelation could be negative [14]. The other modulation methods cause little time jitter.

In a system with $\mathrm{TH}$ codes having ideal autocorrelation and an adequate modulation method, ICI can be reduced to a negligible order. At the same time, ISI also can be reduced as long as the excess multipath delay is smaller than several symbol periods. This benefits from the property of low duty cycle and coincidence autocorrelation. Furthermore, in amplitude-modulated systems especially those with bipolar modulation, the interference from ISI plus ICI can be smaller than that from the pure ICI because of cancellation between antipodal signals.

\section{PERFORMANCE ANALYSIS OF IDEAL RAKE RECEPTION}

In a RAKE receiver with $J$ fingers, when ICI and ISI can be ignored, we should get a "clean" RAKE output and obtain the well-known expressions for instantaneous signal-to-noise ratio (i-SNR):

$$
\gamma_{s}=\rho \frac{E_{s}}{\sigma_{0}^{2}} \frac{\left(\sum_{j=1}^{J} a_{j} g_{j}\right)^{2}}{\sum_{j=1}^{J} g_{j}^{2}},
$$

where $g_{j}$ are the weights of RAKE fingers and depend on combining methods, $\rho$ is the normalized correlation of a single pulse and a template signal in a frame period (and depends on the modulation method), $E_{s}$ is the symbol energy of a transmitted signal, and $E_{s} / \sigma_{0}^{2}$ can be regarded as the symbol SNR of the transmitted signal.

An application of the Cauchy inequality,

$$
\left(\sum_{j=1}^{J} a_{j} g_{j}\right)^{2} \leq \sum_{j=1}^{J} a_{j}^{2} \sum_{j=1}^{J} g_{j}^{2}
$$

which has equality only when $g_{j}=a_{j}$, leads to the wellknown maximal ratio combining (MRC) and the i-SNR becomes:

$$
\gamma_{s, \mathrm{MRC}}=\rho \frac{E_{s}}{\sigma_{0}^{2}} \sum_{j=1}^{J} a_{j}^{2}
$$

Suppose that the receiver knows the polarity of multipath components and combine all fingers' signals using $g_{j}=1 \cdot \operatorname{sgn}\left(a_{j}\right)$, where $\operatorname{sgn}\left(a_{j}\right)$ is the function of getting the sign of $a_{j}$. Then we call this equal gain combining (EGC) and the i-SNR becomes:

$$
\gamma_{s, \mathrm{EGC}}=\rho \frac{E_{s}}{\sigma_{0}^{2}}\left(\sum_{j=1}^{J}\left|a_{j}\right|\right)^{2} / J .
$$

Under the assumption of independence between RAKE fingers, the statistical characteristics of i-SNR can be derived directly now. But for more general performance criteria, such as symbol error probability (SEP), the derivation will involve joint probabilities and $J$-fold integrations and have high computational complexity if treated in a general way [15]. Fortunately, for the lognormal distribution in UWB applications, there are simplifications that make this tractable, as discussed next in Section 4.1.

\subsection{Distribution of the Sum of Lognormal Variables}

We represent the instantaneous SNR $\gamma_{s}$ as the product of a scalar $\lambda$ and a random variable $\alpha, \gamma_{s}=\lambda \alpha$, where $\alpha=\left(\sum_{j=1}^{J}\left|a_{j}\right|\right)^{2}$ for EGC and $\alpha=\sum_{j=1}^{J} a_{j}^{2}$ for MRC. (Note that there are some different definitions of lognormal distribution and a scaling should be applied during transformations between them.)

The basic idea here is to find the pdf of $\alpha$ and substitute the joint pdf involved in performance analysis. There is a general agreement that a sum of independent lognormal random variables can be well approximated by another lognormal random variable when the spread of $\sigma$ in (2) is not too wide $(<30 \mathrm{~dB} \text { in our case })^{2}$ [10-12]. Moreover, this approximation is also applicable to the sum of correlated lognormal variables. So the distribution of $\alpha$ can be treated as lognormal and represented as $\alpha: \Lambda\left(u_{\alpha}, \sigma_{\alpha}^{2}\right)$. Different approaches to find the parameters of the resultant lognormal distribution have been proposed. The Wilkinson approximation (WA) and Schwarz and Yeh approximation (SYA) [11] are most common. The former, which is simpler, first computes the mean and variance of the sum applying the general statistical formulae, and then calculates $u_{\alpha}, \sigma_{\alpha}$ and the pdf through (3) and (4) implicitly; the latter starts in an inverse order; that is, find the mean and variance of the variable $Z=$ $\ln \left(e^{Y 1}+e^{Y 2}\right)$ where $Y_{1}$ and $Y_{2}$ are two Gaussian variables. The obtained mean and variance of $Z$ are $u_{\alpha}$ and $\sigma_{\alpha}$, respectively, if $\alpha=e^{Z}$ where $Z$ is a Gaussian variable implicitly. It is generally believed that SYA can give better accuracy. A simpler and more accurate algorithm for SYA is given in [12]. 
In our opinion, the substance of the arguments is how to find a distribution to best fit the one that already largely deviates from lognormal distribution. The results obtained from WA and SYA can be compared to tell how well the distribution of the sum fits lognormal. The smaller the difference, the better the fit. This is because the two methods derive their results independently while their results are only connected by the mapping of lognormal. When $\sigma$ is fixed and small for all variables, the distribution of the sum highly resembles lognormal and both methods work well. Figure 2 shows $u_{\alpha}$ and $\sigma_{\alpha}$ versus number of fingers $(J)$ in MRC and EGC cases. The curves obtained by different methods overlap totally, indicating complete agreement. The parameters involved in plotting the graph are given in Section 5.

\subsection{Derivation of Explicit Expressions of Performance}

With the known distribution of the random factor in the instantaneous SNR $(\alpha)$ performance of RAKE can be readily evaluated.

The mean and variance of i-SNR can be obtained by:

$$
\begin{aligned}
\mathrm{E}\left[\gamma_{s}\right] & =\lambda \mathrm{E}[\alpha], \\
\operatorname{Var}\left[\gamma_{s}\right]=\lambda^{2} \operatorname{Var}[\alpha] & =\lambda^{2}\left(\mathrm{E}\left[\alpha^{2}\right]-(\mathrm{E}[\alpha])^{2}\right) .
\end{aligned}
$$

The SEP conditional on $\alpha$ is given by:

$$
\operatorname{Pr}(e \mid \alpha)=Q\left(\sqrt{\gamma_{s}}\right)=\frac{1}{\sqrt{2 \pi}} \int_{\sqrt{\gamma_{s}}}^{+\infty} \exp \left(-y^{2} / 2\right) d y .
$$
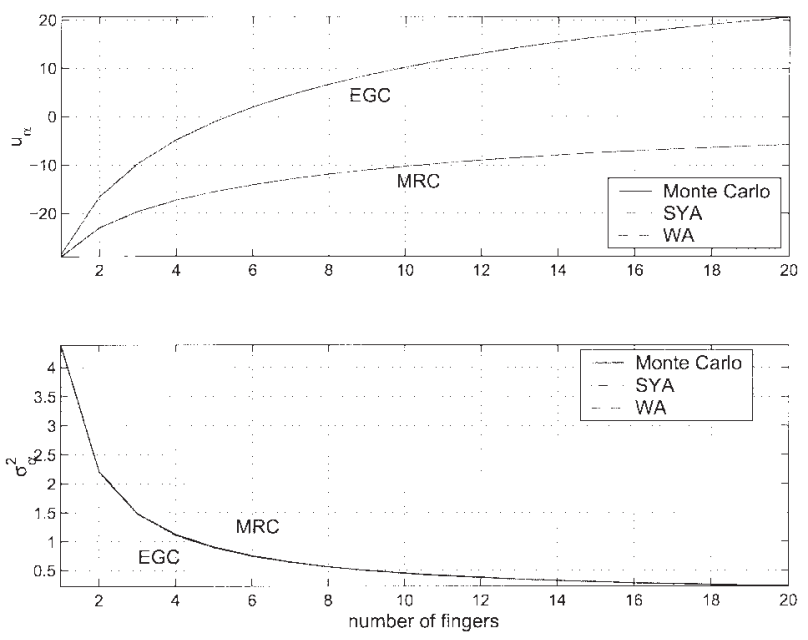

Fig. 2. The $u_{\alpha}$ and $\sigma_{\alpha}^{2}$ for MRC and EGC obtained by the methods of Monte Carlo, SYA, and WA where $\varepsilon_{0}=0.035, \sigma=3.3941 \mathrm{~dB}$, and $\Gamma=$ 4.3. The curves obtained by three methods overlap each other.
Averaging the $\operatorname{Pr}(e / \alpha)$ over $\alpha$, we obtain the mean SEP as:

$$
\operatorname{Pr}(e)=\mathrm{E}_{\alpha}[\operatorname{Pr}(e \mid \alpha)]=\int_{0}^{\infty} Q\left(\sqrt{\gamma_{s}}\right) p(\alpha) d \alpha,
$$

where $p(\alpha)$ is the pdf of $\alpha$.

The mean SEP can be evaluated numerically via Monte Carlo simulation with (14). An alternative way can be used to arrive at an explicit expression, considered next.

Let $\beta=\left(20 \log _{10} \alpha-u_{\alpha}\right) /\left(\sqrt{2} \sigma_{\alpha}\right)$ and exchange $\alpha$ in (14) with $\beta$, yielding:

$\operatorname{Pr}(e)=\frac{1}{\sqrt{\pi}} \int_{-\infty}^{\infty} Q\left(\sqrt{\lambda 10^{\left(\sqrt{2} \sigma_{\alpha} \beta+u_{\alpha}\right) / 20}}\right) \exp \left(-\beta^{2}\right) d \beta$.

In consideration of the weight function $\exp \left(-\beta^{2}\right)$, (15) can be best computed numerically by Hermite-Gauss quadrature [13],

$$
\operatorname{Pr}(e) \simeq \frac{1}{\sqrt{\pi}} \sum_{n=1}^{N_{h}} H_{x_{n}} Q\left(\sqrt{\lambda 10^{\left(\sqrt{2} \sigma_{\alpha} x_{n}+u_{\alpha}\right) / 20}}\right),
$$

where $N_{h}$ is the order of the Hermite polynomial $H_{N h}(\cdot), x_{n}$ and $H_{x n}$ are the zeros (abscissas) and weight factors of $N_{h}$-order Hermite polynomial, respectively. These values are tabulated in many mathematical handbooks (e.g., [13]). In Section 5 , we show $N_{h}=16$ can generate very accurate results where only 16 coefficients are required in the computation.

\section{NUMERICAL EXAMPLES}

For binary PPM, in the case of optimal modulation, $\rho$ is generally in the range of $(0.5,1)$ depending on the pulse used. For bipolar modulation, $\rho$ equals 1 . For convenience, bipolar modulation is adopted to exemplify the effects of RAKE reception below.

For single exponential decay model, parameters are chosen as below:

- Set $\varepsilon_{0}=1 /\left(4 \pi d^{2}\right)$ to account for free space propagation where $d$ is the distance between the transmitter and receiver.

- Instead of simulating a poisson process, the interval of multipath arrival is modelled as fixed. Set $\tau_{\ell}=$ $0.167(\ell-1)$ ns to demonstrate a system in which central-frequency equals $6 \mathrm{GHz}$ and multipathdelay interval equals $0.167 \mathrm{~ns}$.

- $\Gamma=4.3$ and $\sigma=3.3941 \mathrm{~dB}$ corresponds to the ray decay factor and standard deviation of ray lognormal fading term of CM1 in [2], respectively. 
For the dual exponential fading model in [2], we use the data of average power profile of CM1 generated by the program in [2]. The first arrived path is excluded because of the large deviation between it and the succeeding paths.

Figures 3-6 demonstrate the performance based on single exponential decay channel. All figures show EGC has comparable performance with MRC. This alleviates the channel estimation problem greatly.

Figure 3 shows how the mean and variance of i-SNR vary with $J$, Figure 4 demonstrates the variation of mean SEP with $J$ for fixed transmitted SNR $E_{s} / \sigma_{0}^{2}$, and Figure 5 depicts how the mean SEP varies with $E_{s} / \sigma_{0}^{2}$. The numerical computation of mean SEP is based on (16) with $N_{h}=16$. It is clear that the Hermite-Gauss quadrature

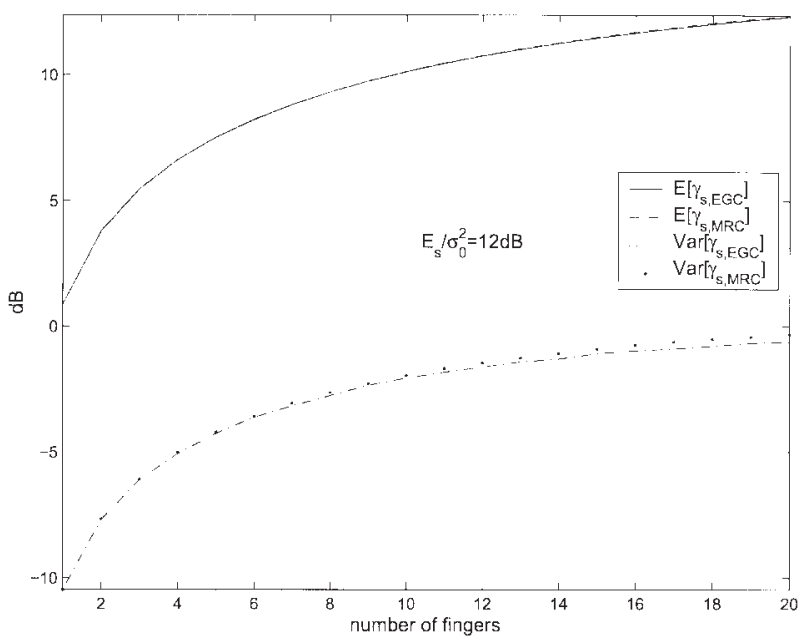

Fig. 3. The mean and variance of i-SNR versus the number of RAKE fingers $(J)$ in EGC and MRC cases when $E_{s} / \sigma_{0}^{2}=12 \mathrm{~dB}, d=1.5 \mathrm{~m}$.

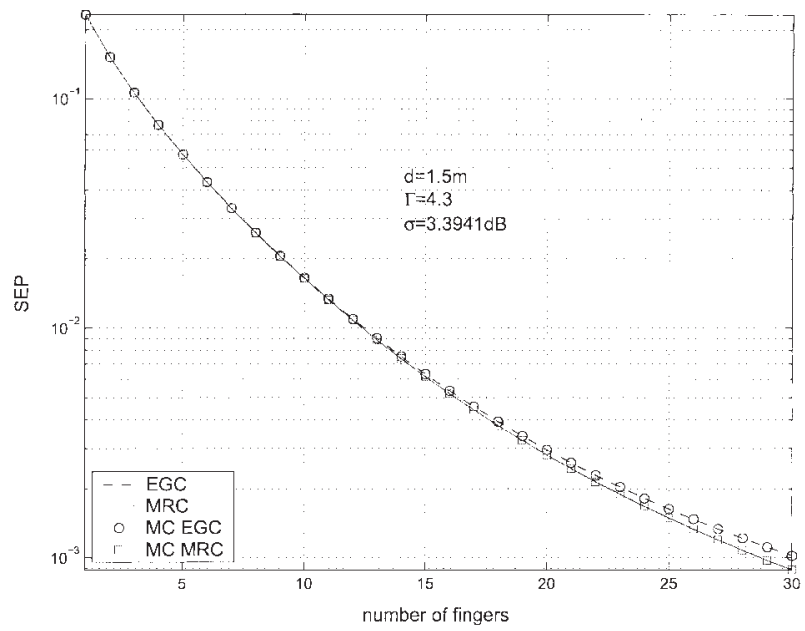

Fig. 4. The mean SEP obtained by (16) and Monte Carlo simulation $(\mathrm{MC})$ versus the number of fingers $(J)$ in EGC and MRC cases when $E_{s} / \sigma_{0}^{2}=12 \mathrm{~dB}, d=1.5 \mathrm{~m}$.

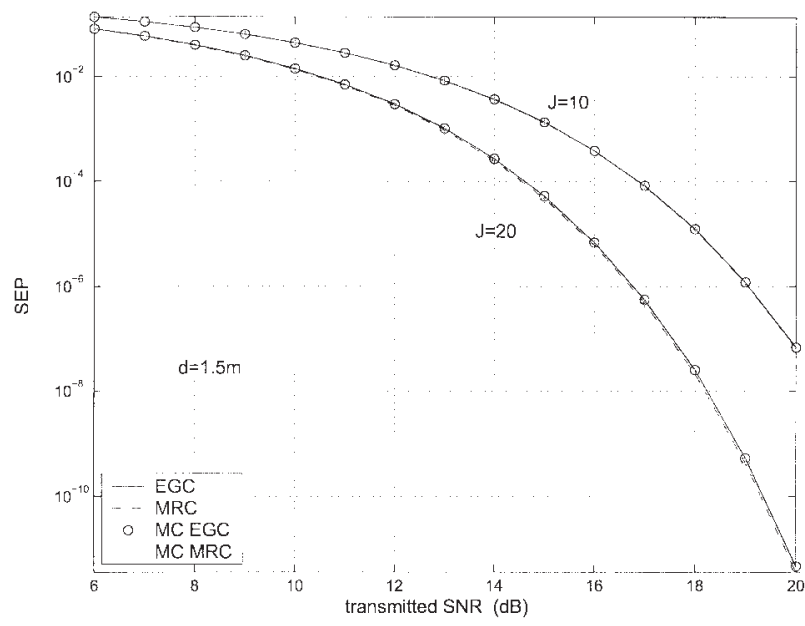

Fig. 5. The mean SEP obtained by (16) and Monte Carlo simulation (MC) versus transmitted SNR $E_{s} / \sigma_{0}^{2}$ in EGC and MRC cases when $d=$ $1.5 \mathrm{~m}, J=10$ and 20 .

generates very accurate results. Comparing Figure 4 and Figure 5, we can find that increasing the transmitted SNR $E_{s} / \sigma_{0}^{2}$ results in much better improvement on the SEP compared to increasing the number of RAKE fingers $J$.

In addition, some experiments on the parameters of the channel model indicate that (i) performance slightly changes and performance difference between MRC and EGC slightly increases with $\sigma$ increasing; (ii) with $\Gamma$ increasing, performance is improved slowly because the mean power of paths decays more slowly and more power can be collected; and (iii) with $\varepsilon_{0}$ increasing, the mean SEP decreases rapidly. Figure 6 shows how the SEP changes with the distance $d$ between transmitter and receiver.

The mean SEP based on the dual exponential fading model in [2] is displayed in Figure 7 to show the relationship between MRC and EGC in three situations. The lines without marks correspond to the theoretical result when applying the average power profile of CM1 in our analysis method. For comparison, the marked lines represent the statistical results directly from the experimental data generated from CM1. Between them, the line marked with circles is based on the original continuous model and the other marked with triangles is based on the sampled discrete model in which the nonzero fingers are chosen. The transmitted SNR here is different for the three cases and is not given because of the unknown gain of the channel models. From Figure 7, we can see that EGC still has comparable performance with MRC in the first two situations, while the difference between them is enlarged in the discrete model. The possible reason is that the discretization of the continuous model causes combination/sum of adjacent paths that have time duration smaller than the sampling time. During each realization, the sum could 


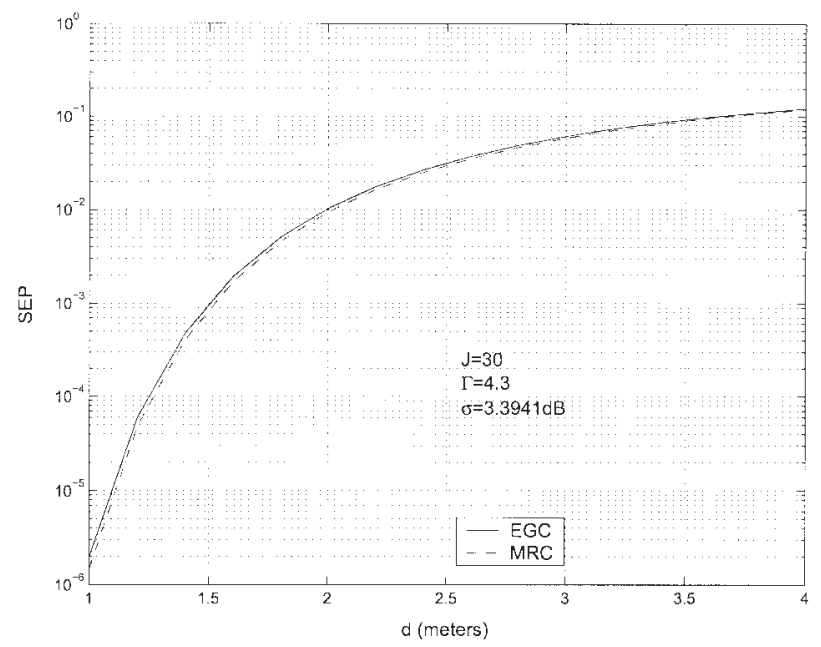

Fig. 6. The mean SEP obtained by (16) versus $d$ in EGC and MRC cases when $E_{s} / \sigma_{0}^{2}=12 \mathrm{~dB}, J=30$.

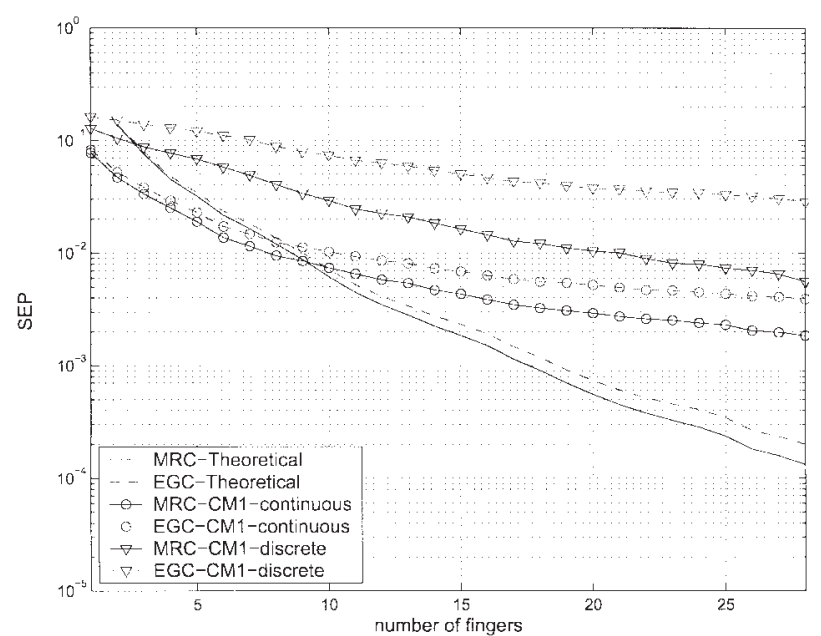

Fig. 7. The mean SEP in EGC and MRC cases when $\sigma=6.4 \mathrm{~dB}$, (a) theoretical results; (b) statistical results based on continuous model in [2]; (c) statistical results based on discrete model in [2].

happen among paths with opposite or same polarity. Then large difference could be arisen among different realizations, which makes it possible that the average power profile could not correctly reflect the statistical property of the channel. This is also the cause of large fluctuation of average power profile. This implies there are limitations of our model on modelling sampled channels.

\section{CONCLUSIONS}

The performance of RAKE reception for UWB is evaluated in a lognormal fading channel. Firstly, we address the fact that in most cases both the property of
TH codes and modulation methods determine whether ICI can be mitigated to a negligible degree. It is emphasized that without further design, the OPPM is not applicable to TH-UWB in the sense of possibly detrimental ICI. With the "clean" output, a simple way is suggested to derive explicit performance expressions for various combining methods based on the fact that the sum of lognormal variables can be well approximated by another lognormal variable under appropriate conditions. Numerical results indicate that RAKE reception can be very effective for UWB, and equal gain combining has comparable performance with maximal ratio combining.

\section{REFERENCES}

1. D. Cassioli, M. Z. Win, F. Vatalaro, and A. F. Molisch, Performance of low-complexity Rake reception in a realistic UWB channel, IEEE International Conference on Communications (ICC), Vol. 2, pp. 763-767, 2002.

2. J. Foerster et al., Channel modeling sub-committee report final, IEEE P802.15 Working Group for Wireless Personal Area Networks (WPANs), IEEE P802.15-02/490r1-SG3a, Feb. 2003.

3. T. Erseghe, Time-hopping patterns derived from permutation sequences for ultra-wideband impulse-radio applications, 6th WSEAS International Conference on Communications, Crete, Vol. 1, pp. 109-115, July 7-14 2002.

4. R. A. Scholtz, P. V. Kumar, and C. J. Corrada-Bravo, Signal design for ultra-wideband radio, Sequences and Their Applications (SETA '01), May 2001.

5. R. Price and P. E. Green, A communication technique for multipath channels, Proceedings of The IRE, Vol. 46, pp. 555-570, 1958.

6. M. Z. Win and R. A. Scholtz, Characterization of ultra-wide bandwidth wireless indoor channels: A communication-theoretic view, IEEE Journal on Selected Areas in Communications, Vol. 20, No. 9, pp. 1613-1627, 2002.

7. R. Jean-Marc Cramer, R. A. Scholtz, and M. Z. Win, Evaluation of an ultra-wide-band propagation channel, IEEE Transactions on Antennas and Propagation, Vol. 50, No. 5, pp. 561-570, 2002.

8. Cassioli M. Z. Win, and A. F. Molisch, The ultra-wide bandwidth indoor channel: From statistical model to simulations, IEEE Journal Selected Areas in Communications, Vol. 20, No. 6, pp. 1247-1257, 2002.

9. F. Ramlrez-Mireles, On the performance of ultra-wide-band signals in Gaussian noise and dense multipath, IEEE Transactions on Vehicular Technology, Vol. 50, No. 1, pp. 244-230, 2001.

10. L. F. Fenton, The sum of log-normal probability distributins in scatter transmission systems, IRE Transactions on Communications Systems, Vol. CS-8, pp. 57-67, 1960.

11. S. C. Schwartz and Y. S. Ych, On the distribution function and moments of power sums with log-normal components, The Bell System Technical Journal, Vol. 61, No. 7, pp. 1441-1462, 1982.

12. C. L. Ho, Calculating the mean and variance of power sums with two log-normal components, IEEE Transactions on Vehicular Technology, Vol. 44, No. 4, pp. 756-762, 1995.

13. A. H. Stroud and D. Secrest, Gaussian Quadrature Formulas, Prentice Hall, Inc., Englewood Cliffs, N. J., 1966.

14. J. Zhang, R. A. Kennedy, and T. D. Abhayapala, New results on the capacity of $M$-ary PPM Ultra Wideband systems, IEEE International Conference on Communications (ICC), May 2003.

15. M. S. Alouini and A. J. Goldsmith, A unified approach for calculating error rates of linearly modulated signals over generalized fading channels, IEEE Transactions on Communications, Vol. 47, No. 9, pp. 1324-1334, 1999. 


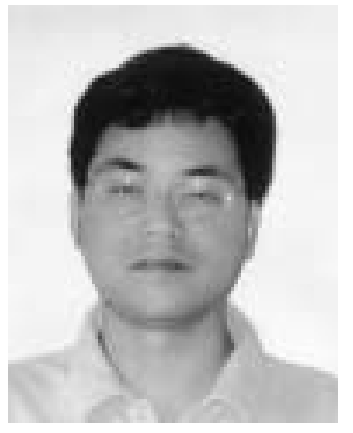

Jian Zhang received the B. S. degree in Telecommunications Engineering from Xi an JiaoTong University, China, in 1996, and M. S. degree from Nanjing University of Posts and Telecommunications, China, in 1999. He is now working toward the Ph.D. degree at the Department of Telecommunications Engineering, Australian National University, Canberra, Australia. From 1999 to 2001, he was employed with Zhongxing Telecom. Corp. (ZTE), China, as a research and development engineer. His current research interests are in the areas of adaptive signal processing and wireless communications, with emphasis on Ultra Wideband systems.

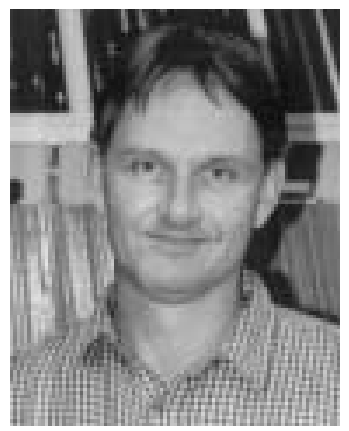

Rod Kennedy has degrees from the University of New South Wales, Australia, University of Newcastle, and the Australian National University. He worked 3 years for CSIRO on the Australia Telescope Project. He has been head of the Department of Telecommunication Engineering, Research School of Information Sciences and Engineering at the Australian National University since 1994. His research interests are in the fields of digital and wireless communications, digital signal processing, and acoustical signal processing. He is currently interim Program Leader for the Wireless Signal Processing Program part of the National ICT Australia (NICTA) research center. He has shared responsibility in supervising about 25 research students, co-authored approximately 180 papers and is on a number of editorial boards for international journals and monograph series.

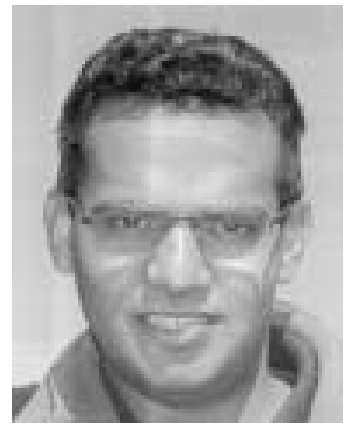

Thushara D. Abhayapala was born in Colombo, Sri Lanka, in 1967. He received the B.E. (hons.) degree in interdisciplinary systems engineering from the Faculty of Engineering and Information Technology, Australian National University (ANU), Canberra, in 1994. He received the Ph.D. degree in telecommunications from the Research School of Information Sciences and Engineering, ANU, in 1999. From 1995 to 1997, he was employed as a Research Engineer at the Arthur C. Clarke Centre for Modern Technologies, Moratuwa, Sri Lanka. From December 1999 to July 2002 he worked as a Research fellow at the Department of Telecommunication Engineering, and as a Lecturer at the Faculty of Engineering and Information Technology, both at ANU. Since July 2002 Dr. Abhayapala has been a Fellow at the Research School of Information Sciences and Engineering, ANU. Currently he also holds an affiliation with the Wireless Signal Processing program, Canberra Research Lab, National ICT Australia (NICTA), Canberra as a Senior Researcher. His research interests are in the areas of space-time signal processing for wireless communication systems, spatiotemporal channel modeling, MIMO capacity analysis, space-time receiver design, equalization, UWB systems, array signal processing, broadband beamforming, and acoustic signal processing. Dr Abhayapala is currently an associate editor for EURASIP Journal on Wireless Communications and Networking. 